\title{
Do Online Resources Give Satisfactory Answers to Questions About Meaning and Phraseology?
}

\author{
Patrick Hanks ${ }^{1}$ and Emma Franklin²( $\left.{ }^{2}\right)$ \\ ${ }^{1}$ University of Wolverhampton, Wulfruna Street, Wolverhampton, WV1 1LY, United Kingodm \\ ${ }^{2}$ Lancaster University, Bailrigg, Lancaster, LA1 4YW, United Kingdom \\ e.franklin@lancaster.ac.uk
}

\begin{abstract}
In this paper we explore some aspects of the differences between printed paper dictionaries and online dictionaries in the ways in which they explain meaning and phraseology. After noting the importance of the lexicon as an inventory of linguistic items and the neglect in both linguistics and lexicography of phraseological aspects of that inventory, we investigate the treatment in online resources of phraseology - in particular, the phrasal verbs wipe out and put down - and we go on to investigate a word, dope, that has undergone some dramatic meaning changes during the 20th century. In the course of discussion, we mention the new availability of corpus evidence and the technique of Corpus Pattern Analysis, which is important for linking phraseology and meaning and distinguishing normal phraseology from rare and unusual phraseology. The online resources that we discuss include Google, the Urban Dictionary (UD), and Wiktionary.
\end{abstract}

Keywords: Online dictionaries $\cdot$ Meaning $\cdot$ Phraseology $\cdot$ Phrasal verbs $\cdot$ Transitivity $\cdot$ Meaning change over time $\cdot$ Corpus pattern analysis

\section{$1 \quad$ Introduction}

As long ago as 1857, the English literary scholar and clergyman Richard Chenevix Trench (subsequently Archbishop of Canterbury) observed that a dictionary is an inventory of the words of a language. Trench pointed out that many words had been missed by the standard dictionaries of his day, including Johnson's. Compiling an inventory is harder than may at first sight appear. Trench's observations "on some deficiencies in our English dictionaries" [6] were a key element in the impetus that prompted the Philological Society to plan a new English dictionary, which many years later was published under a new name as the Oxford English Dictionary (OED). Over 100 years later the great American language teacher Dwight Bolinger [1], in a counterblast to the logical excesses of what was then called transformational grammar, emphasized the importance in language teaching of "getting the words in." But Bolinger went further, in that he started to ask questions concerning what should be said about each word - in a classroom by the teacher or in a dictionary by a lexicographer. An ardent advocate of common sense in language teaching, Bolinger empha- 
sized "the enormous importance of understanding the lexicon for its own sake", not merely as an appendage to the grammar.

Now, in the 21st century, the combined efforts of lexicographers, dialectologists, terminologists, and onomasticians have got us about as close as we can ever hope to get to a complete inventory of the lexicon of English. Now at last the research community can turn its attention to the neglected question of what should be said about each word and in particular the phraseology associated with each word in a language.

Constraints of space and paraphrasing, which bedevilled 19th- and 20th-century dictionaries, are no longer necessarily relevant. We argue that online dictionaries should say something not only about meaning but also about the stereotypical phraseology that characterizes each meaning of each word. Currently available online resources, being inherited from printed dictionaries, tend to say little or nothing about phraseology. They present word meaning in a way that supports the outmoded confusion of natural language with logic that was fashionable in the 19th century, having been inherited from the thinking of logicians such as Leibniz in the early 18th century. To make matters worse, many online resources reflect the traditional belief among old-fashioned lexicographers that the oldest meaning of any word is somehow more literal than its current meaning. The error of this belief can be illustrated with thousands of examples, of which we will give just one: the oldest meaning of the word literal, according to OED, is "of or relating to a letter or letters". But of course the literal meaning of a word or phrase is not the meaning of the letters of which it is composed.

As the use of online dictionaries and other Internet resources becomes ever more commonplace, we must put ourselves in the position of online dictionary users and ask, how useful are such resources? How well do they cater to the needs of English speakers and learners?

\section{$2 \quad$ Trying to Find the Meaning of a Phrasal Verb}

\subsection{Wipe Out}

Imagine you are a dedicated, intermediate-level learner of English. One of your English-speaking friends has shared the news article, Climate Change may Wipe Out Bengal Tigers. You want to know what the headline means. You know what a 'tiger' is, and you know what the verb 'wipe' means: it means to clean something, like your glasses. So wipe + out must mean 'clean out'. But how does something like climate change clean out a tiger? No, that can't be right. "Wipe out", you ask Google. The top result is a YouTube video from a band called Surfaris. The thumbnail is a picture of a surfer being pursued by a gigantic wave. "Okay," you say to yourself, rephrasing the Google search: "wipe out definition". Google Dictionary, by now a familiar sight to you, tells you that wipe out, a phrasal verb of wipe, means "INFORMAL be capsized by a wave while surfing". Capsizing, according to Google Dictionary, is something a boat does. Your mind races; what could be going on here? Are tigers being over- 
whelmed by the floods of climate chaos? Are they climbing into boats? You can't help but be reminded of that famous scene from Life of $P i$, in which a man and a tiger are out at sea.

Thankfully, you find that an alternative sense is listed underneath: "NORTH AMERICAN fall over or off a vehicle". You check and find that, yes, the news story is from a North American website. And climate change is linked to vehicles, after all. This must be it. Google, the most used search engine in the world, would not be wrong about something as straightforward as this. And yet, this explanation is even less plausible than the first. Tigers falling off vehicles? From climate change? Bewildered, you turn to the next result in the list. This time it's from the Cambridge English Dictionary: "Wipe out US INFORMAL - phrasal verb with wipe: to lose control, especially in a vehicle, and have an accident." No further senses are listed. At this point you realize that you must have misunderstood some other part of the headline; what other explanation could there be? You decide to try one more dictionary, just to be sure. Oxford Dictionaries dot com, you type. "The World's Most Trusted Dictionary Provider," it replies. Search term: wipe out. "Wipe out, PHRASAL VERB informal 1. be capsized by a wave while surfing. 1.1 North American fall over or off a vehicle." Apparently, this is where Google gets its dictionary data from.

Baffled, but undeterred, you keep going. It must be a problem with you, the learner. You find the website for the Oxford Advanced Learner's Dictionary (OALD) and type in wipe out. This time, to your surprise, there are three senses available: (1) "wipe out (informal) to fall over, especially when you are doing a sport such as skiing or surfing"; (2) "wipe somebody $\leftrightarrow$ out (informal) to make somebody extremely tired"; and (3) "wipe somebody/something $\leftrightarrow$ out [often passive] to destroy or remove somebody/something completely". Clearly, the best fit here is sense number 3. But what a rare sense this must be! No wonder it was so difficult to find.

As a matter of fact, wiping something out is not rare at all, as we shall see. The problem is a well-known one in English language teaching, namely the moveable direct object of transitive phrasal verbs, which, by the way, is what the double-headed arrow in OALD is trying to explain. Unfortunately, not all intermediate students of English have been taught the difference between transitive and intransitive uses of verbs, and even those who have been taught this point of English grammar may not realize that this is the problem with Google's explanation: it explains only an intransitive meaning of wipe out, whereas the headline about Bengal tigers uses the phrasal verb transitively. By placing a rare intransitive sense before the much more common transitive senses, even the excellent OALD, with its ingenious presentation of the movable direct object, risks confusing the reader. The problem is, what governs what? If a noun phrase is found with a verb and a particle, does the verb govern the noun phrase as a direct object, or does the particle govern it as a prepositional object? For example, a well-known test for phrasal verbs is the example run up a hill vs. run up a bill. It is idiomatic to say he ran up a large bill, which alternates with he ran a large bill up. However, it is not idiomatic in English to say *he ran a large hill up. This is because a large hill is a prepositional object governed by the particle up, whereas $a$ large bill is the direct object governed by the verb and can occur before or after the particle. 


\subsection{Corpus Evidence}

How does all this affect wipe out? The Corpus Pattern Analysis (CPA) project [4], carried out on a sample of the BNC, is being used to create the Pattern Dictionary of English Verbs (PDEV). This is work in progress: as analysis of each verb is completed, an entry is published online (free of charge) at http://pdev.org.uk. Fortunately, a draft entry for wipe has been completed. For a detailed explanation of the concept of "patterns" as well as the full method and theoretical underpinnings of CPA, see [4], also summarized in [3].

PDEV records 10 patterns for the verb wipe; only one of them is for the phrasal verb wipe out. Perhaps surprisingly, the phrasal verb accounts for over $58 \%$ of all uses of the verb wipe; no intransitive uses of this phrasal verb are recorded as patterns in PDEV. The British National Corpus (BNC) was compiled in the 1990s. Perhaps intransitive uses of this phrasal verb have grown in frequency in recent years. An alternative explanation is that intransitive uses with a human subject (surfers or car drivers, as the case may be) are cognitively salient precisely because this is a rare usage. Cognitive salience (i.e. phraseology which is memorable or springs to mind easily) must be distinguished from frequency; the latter is precisely equivalent to social salience. For more on this, see [4].

It is well known that anyone attempting to generalize about the meaning of a word or phrase will likely encounter the problems of granularity: how fine-grained should an explanation be? It is comparatively easy to construct a paraphrase of a whole clause or sentence; much more difficult to construct a set of explanations that will explain all - or even most - future uses of a word or phrase outside of a particular context: contexts provide all sorts of clues to meaning, some of them unexpected and indeed unpredictable. The general sense of wipe out is "to completely remove or destroy". A more fine-grained analysis could distinguish a person or firm being driven into bankruptcy from a species of animal being eliminated from planet Earth. A further distinction could be made between these senses and the more metaphorical sense of causing a person to feel very tired. These distinctions could be expressed as three different patterns for the phrasal verb wipe out, thus:

1. [[Eventuality | Entity 1]] wipe out [[Entity $2 \mid$ Group]] Implicature: [[Eventuality | Entity 1]] completely removes or destroys [[Entity 2 | Group]].

Examples:

If the situation continues unchallenged, not only will local populations of rare dolphins be wiped out, but the threat of extinction looms not far behind. Against that background, the minister seems intent on wiping out salmonella, but only in the egg-laying flocks.

The feeling almost wiped out that terrible year as if it hadn't happened at all.

2. [[Eventuality]] wipe out [[Human | Institution]] 
Implicature: [[Eventuality]] causes [[Human | Institution]] to go bankrupt Example:

If you had what I had, we'd have been totally-ah-bankrupt-well, wiped

out.

The breweries are providing by far the majority of cash flow with which to meet mounting debt payments, running at A $\$ 4$ million a day. A spokesman for Bond Corporation admitted the action could wipe out the entire group.

3. [[Eventuality]] wipe out [[Human $]]$

Implicature: [[Eventuality]] causes [[Human]] to feel very tired.

Example:

People's energy levels vary: they may feel persistently wiped out.

Additionally, an intransitive pattern of this phrasal verb is found, though it was very rare at the time when $\mathrm{BNC}$ was compiled, and is probably not much greater now $(0.2 \%$ of all uses of wipe in the BNC sample; $0.4 \%$ of wipe out; $0.2 \%$ of all uses of wipe in a sample of the Corpus of Contemporary American English (COCA), $0.6 \%$ of wipe out).

4. $[[$ Human $=$ Surfer $]]$ wipe out $[\mathrm{NO}$ OBJ $]$

Implicature: [[Human = Surfer]] is capsized by a wave while surfing

Example:

When I wiped out, it was like going through a car wash without a car.

Pattern 1 above, which Internet dictionaries appear to suggest is unusual, actually accounts for $97 \%$ of a 250 -line BNC sample for the phrasal verb wipe out. If we were to take a more fine-grained approach and split this pattern along the killing/nonkilling divide, we could end up with two patterns: [[Anything]] wipe out [[Human Group | Animal Group]], with the implicature, [[Anything]] kills or causes to be killed all members of [[Human Group | Animal Group]]; and [[Anything]] wipe out [[Abstract Entity | Natural Landscape Feature]], with the implicature, [[Anything]] erases or destroys [[Abstract Entity | Natural Landscape Feature]], with 45\% and $52 \%$ shares of the sample, respectively. There are moral as well as semantic reasons for contemplating this split, but for now let's consider them all as belonging to one pattern.

Patterns 2,3 , and 4 comprise just $2 \%,<1 \%$ and $<1 \%$ of the sample, respectively. Why, then, should a simple internet search using the most powerful search tools available to us produce such misleading results? Should it be down to the user, the possibly unsophisticated learner of English, to know how to differentiate transitive from intransitive uses of verbs? In their study of foreign-language learners' use of online dictionaries, Jin and Deifell [4] conclude that while learners consider such resources to be "essential" to their studies, they also experience a great disparity in the quality of online dictionaries, and generally need to consult several in order to reach a satisfactory answer. Deciding which answer fits best is, as demonstrated above, not always straightforward. Chun [2] notes that the multiple and non-specific definitions offered by online dictionaries can overwhelm lower-level learners. Evidently, there is 
a degree of skill that is required for the user to successfully sort through the piles of conflicting information presented online.

It is important to realize that the ontology used in CPA for analysis of verbs is intended to summarize stereotypes, not all possibilities. [[Group]] may be classed as a subset of [[Entity]], even if counterexamples are found. [[Group]] is specified as a direct object of the phrasal verb wipe out because we find that stereotypically groups of entities such as animals and humans are wiped out. This analysis of stereotypical cases is not invalidated by a handful of counterexamples. On the other hand, if many counterexamples are found, a different semantic type may be selected, as a large number of counterexamples is indicative of a norm that was not originally noticed.

At this point, we should note that bilingual dictionaries often give more information about phraseology than their monolingual counterparts. However, there is still much room for improvement and even simplification. To take just one example, the excellent (printed) Oxford-Hachette (1994) English-French Dictionary offers the following translations for this phrasal verb, which helpfully provides guidance for the prepositional object problem discussed in section 2.1:

wipe out : -- out [sth], -- [sth] out 1 lit (clean) nettoyer [container, cupboard]; 2 Audio, Cin, Comput, Video effacer; 3 fig (cancel) effacer [memory, past]; liquider [debt]; annuler [chances, inflation, gains, losses]; (kill) anéantir [species, enemy, population]; $4^{\circ}$ Sport (defeat) lessiver ${ }^{\circ}$

The references here to the typical direct objects "species, enemy, population", with the gloss (kill), shows that, at least in this context, the correct translation would be anéantir. Should this be prioritized over the other translations offered? Indeed, should all of them be there at all? Unfortunately, current trends in theoretical linguistics, for example in construction grammar, still tend to focus on the theoretically possible on the evidence of introspection, rather than on empirical analysis of actual usage. Corpus evidence is far more plentiful now than it was in the years leading up to publication of Oxford-Hachette in 1994 - and becoming ever more so - so it will be a challenge for future bilingual lexicographers to decide whether to prioritize this particular translation of this particular phrasal verb or even to remove some of the other theoretically possible but unusual translations, if corpus evidence shows them to be vanishingly rare. One would hope that newer dictionaries, as well as online adaptations of older printed dictionaries, would take this newly available data into account.

If we look at the online version of the more recent Oxford-Hachette (2012) Pocket English-French Dictionary, we find just two main senses listed for wipe out:

\section{wipe out}

a. nettoyer «container, cupboard»

b. annuler 〈inflation); anéantir 〈species, enemy, population〉

Similarly, in the online Collins English-French dictionary, we find:

wipe out, separable transitive verb 
1. [debt] effacer

2. [memory $]$ effacer

3. (= destroy) anéantir

Although both of these online entries are considerably slimmed down, we still have the same problem as before in terms of priority and level of generalisation: is the wiping out of inflation, debt or memory to be prioritized over the wiping out of a species? The student translating the "Bengal tigers" headline will once again be led to believe that the "kill" sense of wipe out is one of the rarest, rather than one of the most common. Evidently, both bilingual and monolingual lexicographers could improve the usefulness of online dictionaries by using corpus evidence to not only remove the clutter of rare but theoretically possible information, often lifted from printed dictionaries built on historical principles, but also to order the results in a more empirically defensible way.

\subsection{A Phrasal Verb with Even More Complex Semantics and Phraseology: Put Down}

Let's take a look at another example: the phrasal verb put down. When analysed using the CPA technique, a random sample of the BNC reveals 22 patterns for put down. Patterns and senses, it should be clarified, are not interchangeable concepts; multiple patterns can be used to convey the same sense. Most dictionaries, however, do not list patterns but senses. A Google search for put down definition returns six senses in the default Google Dictionary result. Google Dictionary automatically rephrases the query put down as the transitive put something down. It is not clear why Google decided not to apply the same principle (i.e. automatic rephrasing) to wipe out. Doing so would surely have helped the hypothetical puzzled dictionary user mentioned in an earlier section of this paper.

The six senses listed by Google for put something down are: (1) "Record something in writing" (with a sub-sense of "Make a recording of a piece of music"); (2) "Suppress a rebellion, coup, or riot by force"; (3) "Kill an animal because it is sick, injured, or old"; (4) "Pay a specified sum as a deposit"; (5) "Preserve or store food or wine for future use"; and (6) "Land an aircraft". Combined, these senses represent less than a quarter of the corpus lines that make up the BNC sample for put down. Because Google Dictionary specifies the query put down to refer to put something down, the entry does not include the senses of put someone down, nor is there a crossreference to this entry. This results in a confusing entry for the naïve end user or learner of English.

Evidently, there is an issue with the consistency of entries derived using search algorithms. In the case of wipe out, we received a very specific, and intransitive, sense of the phrasal verb. For put down, we receive this time a transitive, but a similarly restrictive view of the verb's potential meanings. In practice, it turns out, wipe out and put down have similar transitive-to-intransitive ratios; in both cases, the intransitive 
senses (for wipe out, "to be capsized by a wave while surfing" and "to fall off or over a vehicle"; for put down, "(of a plane) to land") constitute $<1 \%$ of their respective BNC samples. Whether or not such findings would bear out using samples from different reference corpora, the fact remains that the same lexicographical principles ought to be applied to all transitive phrasal verb search results.

Returning to put down, let's say that a learner of English encounters the phrase, she put the phone down, and is puzzled by it. So common is this phrase in English that native speakers will find little to be confused about: it means that she ended the telephone conversation. If I say that she put the phone down on me, the normal meaningthe default meaning - is that she ended the phone conversation before I had finished speaking (not that she assaulted me with a mobile phone as a weapon or that she used me as a repository for it). Supposing that the learner knows the very basic - and most common - sense of put down, i.e. "to stop holding something and place it somewhere in the immediate vicinity", they might deduce that the telephone itself was placed somewhere. Given that the use of mobile phones is gradually taking over as the norm of spoken interpersonal communication, in which no physical replacing of a receiver is required, to put down the phone is not necessarily obvious in meaning for a new learner of English. Idiomatic multiword entities such as these are especially important to include in online dictionaries, but they are often absent. In our analysis of a random sample from the BNC, "[[Human 1]] put \{the phone\} down" (with the optional adverbial of "on [[Human 2]]") was found to occur frequently. In fact, it is the fourth most common pattern out of 22 patterns found for put down in the BNC sample.

\section{A Word That Has Undergone Dramatic Changes of Meaning: Dope}

So far, we have considered the role of online dictionaries in the understanding of two commonly used phrasal verbs: wipe out and put down. Let us now take a different example, one that online dictionaries must handle differently. A word whose meaning has changed dramatically over the past century is dope. Apparently originating in America in the $19^{\text {th }}$ century, the noun denoted a kind of thick, gooey porridge or a lubricant, traceable to the Dutch doop 'thick sauce for dipping things in', from doopen 'to dip'. The first dramatic change of meaning, which took place before the end of the $19^{\text {th }}$ century, was to denote a stupid person. This sense has since come to be associated with the use of narcotics, though the history of this word's meaning development is not entirely clear.

In the early $20^{\text {th }}$ century, a further change in meaning took place: dope came to be used as the word for a kind of varnish applied to the canvas fuselage of early aeroplanes. This sense is still found applied to model aeroplanes, which have canvas fuselages and where lightness of weight is all-important. The varnish was notorious as emitting intoxicating fumes, a fact that surely played a role in the development of two modern senses, namely: any of several kinds of stupefying drugs (variously marijua- 
na, opium, or heroin, depending on the particular dialect or region of English); and, as found in both athletics and horse racing, a drug given to athletes, racehorses, and greyhounds, often illegally, either to enhance or inhibit performance. Later in the $20^{\text {th }}$ century, two additional senses developed: 'important information', as in "give me the dope on this development"; and, most recently, a general term of approbation, as in "man, that suit is dope".

Someone using the Internet to decipher the meaning of dope will most likely begin in the usual place: with a Google search for "dope definition". As with all such searches, the default first result is the entry by Google Dictionary, licenced from OxfordDictionaries.com. It is reassuringly comprehensive. For the noun, there are four main senses:

1. INFORMAL a drug taken illegally for recreational purposes, especially cannabis

- a drug given to a racehorse or greyhound to inhibit or enhance its performance

- a drug taken by an athlete to improve performance

2. INFORMAL a stupid person

3. INFORMAL information about a subject, especially if not generally known.

4. a varnish formerly applied to fabric surfaces of aircraft to strengthen them and keep them airtight

- a thick liquid used as a lubricant

- a substance added to petrol to increase its effectiveness.

For the verb, three main senses are given:

1. administer drugs to (a racehorse, greyhound, or athlete) in order to inhibit or enhance sporting performance

- INFORMAL, be heavily under the influence of drugs, typically illegal ones

- $\quad$ treat (food or drink) with drugs

- $\quad$ add drugs to, tamper with, adulterate, contaminate

- INFORMAL, DATED regularly take illegal drugs

2. smear or cover with varnish or other thick liquid

3. ELECTRONICS add an impurity to (a semiconductor) to produce a desired electrical characteristic.

For the adjective just one sense is given:

1. very good.

Finally, an explanation of the phrasal verb dope something out is listed, with the meaning, "work out something".

Unlike our previous examples, in which the online dictionary entries were found to be inadequate, so that multiple resources needed to be consulted in order to find a 
relevant definition, this first available entry encapsulates every known meaning of the word dope.

\subsection{Urban Dictionary}

Our next Google result is not one of the usual contenders, such as Cambridge English Dictionary, Oxford Dictionaries, or Merriam Webster, but instead the Urban Dictionary, a website dedicated to defining slang terms not found in standard dictionaries. That this website ranks so highly in the search results for "dope definition" is a reflection of the heavily informal nature of dope.

Urban Dictionary (UD) is a collection of crowd-sourced definitions for terms that are typically not found in standard dictionaries or are controversial in some way. Entries are submitted by members of the public and can be 'upvoted' or 'downvoted' by fellow users, meaning that definition search results are in fact rankings of candidate entries for a given term based on the upvote-to-downvote ratio. Rather than being professionally produced by lexicographers, these are written by lay people, often featuring slang, jocular language. UD features 176 submitted entries for dope, suggesting that there is a degree of disagreement over meanings and nuance. The "top" entry for dope reads as follows:

Saying something is cool. Most heard in big cities. Or, a drug. (Ex. Cocane [sic]/Mary J/ Dope)".

Examples of usage are provided, presumably invented by contributors, as with almost all UD definitions: "'Yo that new shirt is dope!' Or 'Look! Some guy is selling dope!"'. This has been voted the "top" definition presumably because users find it the most useful or, in their experience, accurate.

In the second-highest-ranking UD entry, dope is defined as "Old people definition: Marijuana. Southern definition: Meth. Northern definition Heroin. Also dope can mean awesome." This has been corroborated (upvoted) by 823 users, who seem to agree with this evaluation of the age and regional differences in the use of the term. The third entry, voted for by 18,757 users, also includes expressions of opinion, possibly based on observations of usage: "People who do not do drugs call Marajuanna [sic] Dope. People who do Marajuanna [sic] call Heroin Dope. Word has also been used to describe how good somthing [sic] is."

Despite the unconventional spelling and the absence of lexicographical etiquette e.g. word class, register information, etymology and so on - these entries are both informative and interesting; they are also evidently popular, no doubt because they strike at the heart of the issue: dope is not a neutral term. Despite its positive connotations as an adjective, it tends to have a negative connotation when used as a noun. Entry number 3 even alludes to a kind of moral hierarchy of drug users: those who use no drugs at all, those who use cannabis, and those who use heroin. 
How reliable are these entries? In a sample of the Contemporary Corpus of American English (COCA) for the lemma DOPE, we found that $73.5 \%$ was constituted by instances of the noun dope, denoting a stupid person. $9.2 \%$ of the sample was made up of examples of the positive adjective dope. The verb dope, perhaps surprisingly, has a very low frequency, constituting just $2.2 \%$ of the sample. In a sample taken from the $\mathrm{BNC}$, for comparison, $75.5 \%$ of concordance lines featured the noun that refers to a drug; $6.1 \%$ referenced a stupid person; $11.2 \%$ featured the use of dope as a transitive verb; and $5.1 \%$ of the sample was concerned with the electronics sense of the term, as explained in the Google/Oxford definition above. The use of dope as an adjective is negligible in the BNC sample at just $1 \%$ - but it is slightly more frequent in COCA, a fact that suggests that this is a typically American use of the word.

\section{$4 \quad$ Wiktionary}

Next we asked ourselves, how good is Wiktionary as an online resource for questions about English phraseology and meaning? Like most online resources these days, it is free of charge, but that does not necessarily mean that it is any good.

We looked at the Wiktionary entries for the three cases that we have been studying - the phrasal verbs wipe out and put down, and the word dope, a word that is used both as a noun and a verb, and in recent years has also come to be used in slang as an adjective.

\subsection{Phrasal Verbs: Wipe Out and Put Down}

The first thing to say is that Wiktionary does not offer systematic cross-references from base verbs to phrasal verbs. In other words, the user has to already know that wipe out, put down, and many similar expressions are phrasal verbs in order to find the most common uses and meanings of these verbs, i.e. wipe and put. Wiktionary's failure to point the reader from the base verb to the phrasal verb means that it cannot be regarded as a reliable aid for intermediate students of English. It seems to be symptomatic of a false belief by the proprietors and software engineers of Wiktionary that the vocabulary of a natural language is more stable than it really is and that what online users of lexical resources really want are: a) foreign-language equivalents of lexical items and b) links to rare and unusual words. This supposition is borne out by the usual set of cross-references that are actually given at the verb put, including forthput, input, puttable, and the obsolete northern English dialect inflected form putten. In view of this bias, it is not surprising that, whereas Wikipedia has been enthusiastically adopted by almost everyone except a few pedants, its sister product, Wiktionary, is very much less popular. 
Corpus evidence shows that by far the most common use of the verb wipe is in the phrasal verb wipe out, in the sense: "to destroy (a large number of people or things); to obliterate." A similar but more complicated story can be told about put down.

Having said that, we hasten to add that any readers who do manage to find the entries for the phrasal verbs are likely to find an excellent set of definitions - provided that the phrasal verb in question has not changed its meaning during the past 60 years. The reason for this is that most of Wiktionary's definitions are taken from an old edition (out of copyright) of Merriam-Webster's Collegiate Dictionary, which was compiled by professional lexicographers in the 1950s and 60s. Some more recent additions are accurate too (as in the case of sense 4 of wipe out, below). However, all too often Wiktionary's policy of allowing anybody to say anything leads to unsatisfactory results, as we shall see when we look at the entry for dope.

The Wiktionary entry for wipe out reads as follows:

1. (idiomatic) To destroy (a large number of people or things); to obliterate.

2. To physically erase something written.

3. To do away with; to cause to disappear.

4. (intransitive) To crash, fall over (especially in board sports such as surfing, skateboarding etc.)

As with wipe out, the entry for put down does not state explicitly that this is a phrasal verb, the base of which is the verb put. Instead, there are useful links to entries for put down as, put down for, and put down to, and the noun form put-down, meaning "an insult or barb; a snide or demeaning remark".

The entry for put down is comprehensive and well defined:

1. Used other than with a figurative or idiomatic meaning: see put, down. Why don't you put down your briefcase and stay awhile?

2. (idiomatic) To insult, belittle, or demean. They frequently put down their little sister for walking slowly.

3. (of money as deposit) To pay. We put down a $\$ 1,000$ deposit.

4. To halt, eliminate, stop, or squelch, often by force. The government quickly put down the insurrection.

5. (euphemistic) To euthanize (an animal). Rex was in so much pain, they had to put him down.

6. To write (something). Put down the first thing you think of on this piece of paper.

7. (of a telephone) To terminate a call; to hang up. Don't put the phone down. I want a quick word with him, too.

8. To add a name to a list. I've put myself down for the new Spanish conversation course.

9. To make prices, or taxes, lower. $B P$ are putting petrol and diesel down in what could be the start of a price war.

10. (idiomatic) To place a baby somewhere to sleep. 
I had just put Mary down when you rang. So now she's crying again.

11. (idiomatic, of an aircraft) To land.

The pilot managed to put down in a nearby farm field.

12. (idiomatic) To drop someone off, or let them out of a vehicle.

The taxi put him down outside the hotel.

13. (idiomatic) To cease, temporarily or permanently, reading (a book). I was unable to put down The Stand: it was that exciting.

Our only serious criticism here is that the wording of sense 7 here is misleading. "Of a telephone" implies that putting down is something that a telephone does, rather than something that a person does to a telephone. This would certainly complicate things for our hypothetical learner who is trying to decode she put the phone down on me (see Section 2.3).

\subsection{Dope}

The Wiktionary entry for dope is clearly based on an old dictionary that is out of copyright, evidently an edition of Merriam-Webster's Collegiate. Here, the unsatisfactory nature of the policy of augmenting an old dictionary by crowdsourcing is evident. The definitions for the noun senses of this word are as follows:

1. Any viscous liquid or paste, such as a lubricant, used in preparing a surface.

2. (uncountable) An absorbent material used to hold a liquid.

3. (uncountable, aeronautics) Any varnish used to coat a part, such as an airplane wing or a hot-air balloon in order to waterproof, strengthen, etc.

4. (uncountable, slang) Any illicit or narcotic drug that produces euphoria or satisfies an addiction; particularly heroin.

5. (uncountable, slang) Information, usually from an inside source, originally in horse racing and other sports.

What's the latest dope on the stock market?

6. (uncountable, firearms) Ballistic data on previously fired rounds, used to calculate the required hold over a target.

7. (countable, slang) A stupid person.

8. (US, Ohio) dessert topping

Wiktionary fails to say that senses 4 and 7 (and possibly 5) are the only ones that are in current usage, although sense 3 (a relic of the early days of aeronautics, when airplane fuselages were made of cloth, for the sake of lightness, then strengthened with varnish) still survives among makers of model planes. The label "aeronautics" is misleading, to say the least. Dope has not been an active term in mainstream aeronautics since before the Second World War.

Sense 6 appears to be a subsense of sense 5, presumably added by somebody with a specialist interest in firearms. It seems that no attention has been given to lexicographical principle getting the right level of generalization. 
Sense 8 appears to have been added by someone living in Ohio, representing an unusual local sense of the word.

Worse is to follow. Wiktionary aims to be multilingual, but its translation equivalents are handled without linguistic sensitivity. To take just one example, the first French translation equivalent for sense 1 of dope is said to be patine, but this is glossed back into English as "patina, an oxidation like on bronze or similar effect."

The very principle that has made Wikipedia such a resounding success - an open forum in which anybody can contribute an article on subjects of which they have specialist knowledge - has contributed to the failure of Wiktionary. It is not a reliable resource. By attempting to satisfy everybody's linguistic needs, Wiktionary succeeds in satisfying nobody's.

The "welcome page" or blurb proudly announces, "Wiktionary is a multilingual free dictionary, being written collaboratively on this website by people from around the world. Entries may be edited by anyone!" In this paper, we have suggested that this policy is responsible for some bad results.

\section{Conclusions}

How reliable are these definitions? Where dope is concerned, analyses of samples from the BNC and the Corpus of Contemporary American English (COCA) suggested that most of the important senses are well covered by Oxford/Google at least. The same is true, up to a point, of the phrasal verbs, though the cross-referencing policy is in serious need of attention. The most common sense of wipe out, for example, is discoverable in online dictionaries but only for those who are determined to keep digging. Online dictionaries such as Oxford Dictionaries, which is now the default option offered by Google, do a pretty good job of explaining the contemporary and historical meaning of words in isolation, all things considered. However, this paper has drawn attention to two areas in which further work is desirable.

We suggest that Oxford and Google between them have not taken sufficient account of the difference between a printed dictionary and an electronic resource. This particularly affects questions about phraseology and in the case of phrasal verbs such as wipe something out or wipe someone out, results can be extremely confusing for a user who does not already know the meaning. On the printed page, wipe something out and wipe someone out can be clearly seen as part of the entry for wipe, but in an online resource these transitive phrasal verbs are hidden from view and may never be found by naïve users.

A first step towards remedying this deficiency would be for the software engineers in Oxford or Silicon Valley to introduce cross-references systematically for subentries that are not immediately visible in an online resource. More ambitiously, it would be desirable to distinguish the many rare, obsolete, and obsolescent senses of words from those that are still in common use today. This could only be done with reasonably reliable results by systematically sampling usage in a large corpus. Empirical analysis 
of phraseology by Corpus Pattern Analysis $[3,4]$ is necessary to distinguish normal, conventional uses words from unusual uses. Unusual uses include senses that are no longer current, but also freshly created metaphors and other exploitations of the normal patterns of word use. (It should be noted here that there are two kinds of figurative language, as found using CPA and detailed in [4], including metaphors: freshly created exploitations of normal phraseology; and conventional metaphors, which are secondary norms rather than examples of linguistic creativity.) Understanding the differences between norms and exploitations is key to providing relevant information on word meanings.

We also took a brief look at the Urban Dictionary and concluded that, while this is not to be taken seriously as a work of monolingual lexicography (an inventory of the words of English and their meanings) it allows people interested in contemporary usage (not professional lexicographers) to present some very interesting observations. It could even be seen as a beneficial application of the principle of crowd-sourcing. Unfortunately, the same cannot be said for Wiktionary, which falls between two stools. On the one hand it is basically an old dictionary, which was compiled in the $1950 \mathrm{~s}$ or 60s. A policy of crowd-sourcing allows anyone to add anything to this base, often with unsatisfactory results, which are no help to anyone. We contrasted this with Wikipedia, where the crowd-sourcing principle allows people with specialist knowledge to contribute their expertise to the benefit of the whole community.

While the cases explored in this paper are necessarily few, they have demonstrated intrinsic problems with online lexicographic resources. Whether one begins with a Google search for "[word] definition", or whether one goes direct to the source, such as OxfordDictionaries.com or another online resource, the user will most likely be required to have relatively sophisticated linguistic knowledge to successfully navigate the results. Traditional printed dictionaries suffer with their own problems, but given the massive potential of the Web in terms of availability and richness of data, crossreferencing capability and lack of space constraints, online resources clearly have much room for improvement. 


\section{References}

1. Bolinger, D. 'Getting the Words In.' In R. I. McDavid and A. R. Duckert (eds.) Lexicography in English. New York Academy of Sciences (1973).

2. Chun, D. M. 'L2 reading on the web: Strategies for accessing information in hypermedia'. Computer Assisted Language Learning, 14(5), pp. 367-403 (2001).

3. Hanks, P. 'Corpus Pattern Analysis'. In G. Williams and S. Vessier (eds.) Proceedings of the Eleventh EURALEX International Congress, EURALEX 2004, Vol. 1, Lorient, France, July 6-10. Université de Bretagne-Sud (2004).

4. Hanks, P. Lexical Analysis. Cambridge, MA: MIT Press (2013).

5. Jin, L. and Deifell, E. 'Foreign language learners' use and perception of online dictionaries: A survey study'. Journal of Online Learning and Teaching, 9(4), pp. 515-533 (2013).

6. Trench, R. C. 'On some Deficiencies in our English Dictionaries'. Transactions of the Philological Society, 4(2), pp. 1-70 (1857). 